\title{
ANTENATAL CARE AND NEONATAL MORTALITY AND MORBIDITY IN THREE HOSPITALS OF PUNJAB, PAKISTAN
}

\author{
Rizwana Akbar, Naila Azam*, Fatima Ali Raza Mughal*, Mahmood Ur Rahman**, Ahmed Tariq"**, Maira Wajahat ${ }^{* * * *}$ \\ Combined Military Hospital/ National University of Medical Sciences (NUMS) Rawalpindi Pakistan, *Foundation University Medical College Islamabad \\ Pakistan, **HITEC Institute of Medical Sciences, Taxilla/National University of Medical Sciences (NUMS) Pakistan, ***Army Medical College/National University \\ of Medical Sciences (NUMS) Rawalpindi Pakistan, ${ }^{* * * *}$ Pak Emirates Military Hospital/National University of Medical Sciences (NUMS) Rawalpindi Pakistan
}

\section{ABSTRACT}

Objective: To find out the association between neonatal morbidity \& mortality and the number of ante-natal visits of the mother of the newborn.

Study Design: Comparative cross-sectional study.

Place and Duration of Study: Neonatal Intensive Care Units (NICUs) of three military hospitals of the Punjab province, from Jan to Mar 2019.

Methodology: Data of all newborns (total of 3065 neonates) delivered in selected Military Hospitals, and all newborns admitted to Neonatal Intensive care units, of Military Hospitals born in same hospital (in born) and born outside the Military Hospital (out born) during this period along with their mothers was collected. Neonatal morbidity and mortality in the selected hospitals was recorded. Mothers were classified as booked and unbooked according to the number of antenatal care visits. Data was analyzed by Statistical Packages for Social Science (SPSS) version 20.

Results: Neonatal morbidity including prematurity, low birth weight, birth asphyxia, neonatal sepsis, pneumonia, diarrhoea, congenital malformation, and fits was significantly less $(p$-value $<0.001)$ in children whose mothers had at least 4 or more antenatal visits (booked) than those newborns whose mothers had less than 4 antenatal visits (unbooked). The neonatal mortality in booked mothers was less than half of that in unbooked mothers.

Conclusion: Booked mothers had better outcomes in terms of neonatal health and survival, and the neonatal health and survival was strongly correlated with the number of antenatal visits of the mother.

Keywords: Antenatal visits, Neonatal mortality, Neonatal morbidity, Prevention.

This is an Open Access article distributed under the terms of the Creative Commons Attribution License (http://creativecommons.org/licenses/by/4.0), which permits unrestricted use, distribution, and reproduction in any medium, provided the original work is properly cited.

\section{INTRODUCTION}

Every year, millions of children under 5 years of age die of preventable diseases ${ }^{1}$. These deaths reflect the limited access of children to basic health needs, such as vaccination, treatment of infectious diseases, adequate nutrition and sanitation ${ }^{2}$. According to the UN Millennium Development Goals3, Goal 4 was to reduce Child Mortality Rate which could not be met without substantial reduction in neonatal mortality especially in the developing countries like Pakistan ${ }^{4,5}$.

In September 2015, with the end of the era of the Millennium Development Goals, the international community agreed on a new framework, the Sustainable Development Goals ${ }^{6}$ (SDGs). Goal 3 in the SDGs is to ensure healthy lives i.e. "good health and wellbeing and promote wellbeing for all at all ages". The SDG target number 3.2 for child mortality represented a renewed commitment to the world's children ${ }^{7}$. The goal is to end preventable deaths of newborns and children under 5 years of age by the end of 2030. It aims to

Correspondence: Dr Rizwana Akbar, Neonatal Intensive Care Units, Combined Military Hospital Rawalpindi Pakistan

Received: 24 Aug 2020; revised received: 28 Dec 2020; accepted: 31 Dec 2020 reduce neonatal mortality to as low as 12 deaths per thousand live births and under 5 mortality to at least as low as 25 deaths per thousand live births" 8 .

In 2017, out of 5.4 million annual deaths of children below 5 years of age, about 2.5 million were neonates ${ }^{4}$. Trends show that the proportion of child deaths that are in the neonatal period has increased in all regions of the world and globally was $41 \%$ in $2010^{8}$, whereas, in 2017, this has increased to $46.2 \%$ of all under 5 deaths ${ }^{4}$. The main direct causes of neonatal death are estimated to be preterm birth, Asphyxia, respiratory distress, severe infections (sepsis), Pneumonia, and congenital abnormalities ${ }^{9}$.

Neonatal mortality, Infant Mortality and Under 5 years mortality rates are important health indicators of a country ${ }^{10}$. There has been a steady decline in the under 5 year mortality rate in Pakistan from 138/1000 live births in 1990 to $74.9 / 1000$ live births in 2017, a $45.72 \%$ decline. The neonatal mortality rate of 44.2 per 1000 live births of Pakistan in 2017 accounted for $59.01 \%$ of under 5 mortality rate in 2017. There has been a downward trend in the infant mortality rate as well, a total decline of 32.9\% between 1990 and 2017, from $91 / 1000$ live births to $61 / 1000$ live births ${ }^{4}$. 
As $74 \%$ of neonatal deaths occur in the first week of life, they are attributable to maternal causes as well, which are preventable. Quality antenatal care with an appropriate number of visits, appropriately distributed during pregnancy can help detect the morbidities during pregnancy and thus help to reduce the complications during pregnancy and labour ${ }^{9}$. This can improve outcomes of pregnancy and thus reduce preventable neonatal deaths.

This study aimed to find the difference in neonatal morbidity and mortality among booked and unbooked mothers for antenatal care.

\section{METHODOLOGY}

This was a comparative cross-sectional study. Data was collected from the Neonatal Intensive Care Units (NICUs) of military hospitals of Rawalpindi, Jhelum and Mangla and Consulting National data from Pakistan Demographic Health Survey (PDHS) 2017-1811.

Data Collection was completed in three Months from January to March 2019. The study population included newborns who were delivered in the three selected hospitals and all newborns admitted in NICUs of pediatric departments of the three hospitals delivered within (inborn) or outside (outborn) these hospitals. Informed consent was taken from their mothers/ next of kin. Newborns of mothers/next of kin who did not give written informed consent and newborns who did not reach the selected military hospitals were excluded from the study.

Neonatal morbidity and mortality in the selected hospitals was recorded. Mothers were classified as booked and unbooked according to the number of antenatal care visits. At least 4 antenatal visits were recommended for mothers in the focused ANC (FANC) model by WHO in 2002. However according to WHO, since November 2016, "Antenatal care models with a minimum of eight contacts were recommended to reduce perinatal mortality and improve women's experience of care". In this study we considered mothers with 4 or more visits as booked for antenatal care. If a mother had 3 antenatal visits she was considered as partially booked. If a mother had less than 2 visits or no antenatal care visits she was considered unbooked. However for inference of results mothers with 4 or more ANC visits were considered as booked, those with less than 4 visits were considered as unbooked.

This study was conducted after approval from the Ethics Review Board of Armed Forces Postgraduate
Medical Institute. Permission was sought from the Commanding Officers / Commandants of the respective hospitals for the collection of data. Coding was used to guarantee the confidentiality of data/ information. No monetary compensation was given to the study participants.

Data was analyzed by Statistical Packages for Social Science (SPSS) version 20. The responses of the questionnaires were coded and scored to facilitate the entry of data. Quantitative variables were reported as Mean \pm SD (Standard Deviation) while categorical variables were reported as frequencies and percentages. The results of the study have been presented in the form of tables in the results section. For inferential statistics, chi-square test was applied to find an association between variables. The confidence interval was taken as $95 \%$ and a margin of error 5\%, $p$-value $\leq 0.05$ was considered statistically significant.

\section{RESULTS}

A total of 3065 neonates including inborn and outborn babies were included in the study. 2382 were from Rawalpindi, 351 were from Jhelum and 332 were from Mangla. Out of a total of 3065 neonates, 1532 (50\%) were female and 1533 (50\%) were male.

Mostly all newborns were full term. Mothers of 585 (19.1\%) were unbooked, they had less than 2 visits, mothers of $2198(71.7 \%)$ were partially booked, they had 3 visits, and mothers of $282(9.2 \%)$ had 4 or more ANC visits and were booked (figure).

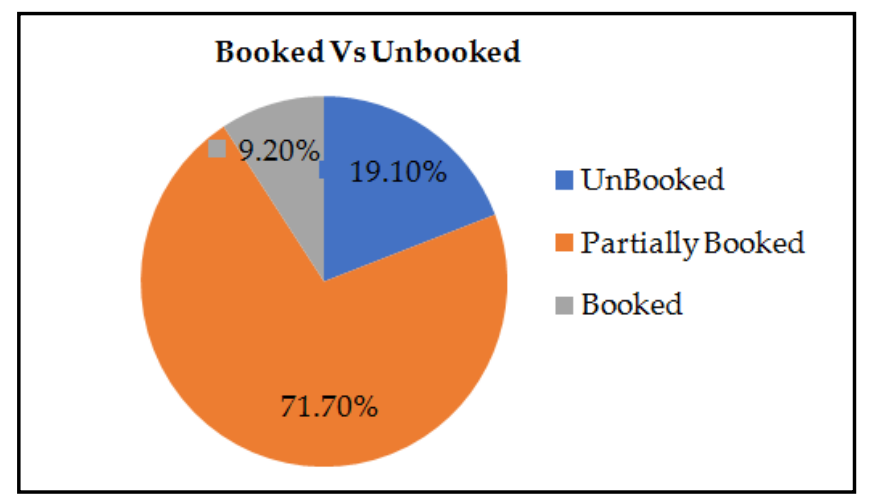

Figure: Booked vs unbooked mothers.

The newborns of mothers who had 4 or more ANC visits (booked) had significantly lower morbidity as compared to newborns of mothers who had $<4$ ANC visits (unbooked) during pregnancy. There were strong statistically significant differences between prematurity $(p=0.001)$, low birth weight $(p=0.001)$, Birth asphyxia $(p=0.001)$, neonatal sepsis $(p=0.001)$, pneumonia $(p=0.001)$, diarrhoea $(p=0.019)$, congenital mal- 
formation $(p=0.01)$, fits $(p=0.001)$, post-term $(0.001)$ in booked and unbooked status of mothers (table-I).

Out of 3065 neonates, 153 (4.99\%) neonates died. This shows a mortality rate of 49.9 deaths per 1000 live births. In Rawalpindi there were 124 (5.3\%) neonatal deaths, in Jhelum there were 13 (3.7)\% neonatal deaths while in Mangla there were $16(4.8 \%)$ neonatal deaths. Mortality was significantly higher in children of unbooked mothers compared to booked and partially booked mothers.

\section{DISCUSSION}

The objectives of the study were to find out the frequency of neonatal mortality and morbidity in Neonatal Intensive Care Units (NICUs) of pediatric departments of three military hospitals of Mangla, Rawalpindi and Jehlum and compare the neonatal mortality and morbidity in cases where mothers were booked for antenatal care with those of unbooked mothers in the same setting, thus to study the impact of antenatal care on neonatal morbidity and mortality.

Table-I: Association of neonatal morbidity with booked and unbooked status of mothers.

\begin{tabular}{|c|c|c|c|c|}
\hline Neonatal Morbidity & Un Booked & Partially Booked & Booked & $p$-value \\
\hline Prematurity & $88(22.7)$ & $287(74)$ & $13(3.4)$ & 0.001 \\
\hline Low birth weigh & $82(35.5)$ & $133(57.6)$ & $16(6.4)$ & 0.001 \\
\hline Birth asphyxia & $105(27.8)$ & $160(53.2)$ & $36(12)$ & 0.001 \\
\hline Neonatal sepsis & $59(27.8)$ & $127(59.9)$ & $26(12.3)$ & 0.001 \\
\hline Pneumonia & $31(20.4)$ & $91(59.9)$ & $30(19.7)$ & 0.001 \\
\hline Diarrhoea & $17(30.4)$ & $26(47.3)$ & $12(21.8)$ & 0.019 \\
\hline Congenital malformations & $17(54.8)$ & $13(41.9)$ & $1(3.2)$ & 0.001 \\
\hline Jaundice & $36(17.9)$ & $149(74.1)$ & $16(8)$ & 0.963 \\
\hline Fits & $37(39.4)$ & $52(55.3)$ & $5(5.3)$ & 0.001 \\
\hline Post term & $4(57.1)$ & $3(42.4)$ & - & 0.007 \\
\hline Others & $38(44.7)$ & $22(25.9)$ & $25(29.4)$ & 0.001 \\
\hline
\end{tabular}

Table-II: Neonatal Mortality Rate in booked and unbooked mothers of newborns admitted in Neonatal Intensive Care Units of the selected military hospitals. Booking Status of Mothers $\quad$ Neonatal Mortality Rates \begin{tabular}{|l|l}
\hline Booked & 21/1000 live births \\
\hline
\end{tabular} \begin{tabular}{|l|l}
\hline Partially Booked & $49.9 / 1000$ live births \\
\hline
\end{tabular} \begin{tabular}{l|l}
\hline Unbooked & $87.6 / 1000$ live births \\
\hline
\end{tabular}

Table-III: Frequency of neonatal morbidity in cases of neonatal mortality.

\begin{tabular}{l|c}
\hline & n (\%) \\
\hline Prematurity & $112(61.9)$ \\
\hline Low birth weigh & $72(39.8)$ \\
\hline Birth asphyxia & $40(22.1)$ \\
\hline Neonatal sepsis & $66(36.5)$ \\
\hline Pneumonia & $16(8.8)$ \\
\hline Diarrhoea & $7(3.9)$ \\
\hline Congenital malformations & $9(5)$ \\
\hline Jaundice & $37(20.4)$ \\
\hline Fits & $53(29.3)$ \\
\hline Post term & $1(0.6)$ \\
\hline Others & $11(0.6)$ \\
\hline
\end{tabular}

Table-IV: Association of neonatal mortality with booked and unbooked status of mothers of newborns admitted in Neonatal Intensive Care Units of the selected hospitals.

\begin{tabular}{l|c|c|c|c}
$\begin{array}{l}\text { Neonatal } \\
\text { Mortality }\end{array}$ & Unbooked & $\begin{array}{c}\text { Partially } \\
\text { booked }\end{array}$ & Booked & $\begin{array}{c}p \text { - } \\
\text { value }\end{array}$ \\
\hline Yes & $22(8.7)$ & $124(5.1)$ & $7(2.1)$ & 0.023 \\
\hline No & $229(91.3)$ & $2369(94.9)$ & $315(97.9)$ & \\
\hline
\end{tabular}

The study showed that there was a highly significant association between the number of antenatal visits of the mother and the mortality and morbidity of newborns. Mothers with 4 or more antenatal visits had much better pregnancy outcomes. There was a statistically significant difference in neonatal mortality and morbidity in booked and unbooked mothers. The neonatal mortality in booked mothers was less than one half of that in unbooked mothers.

Out of 3065 newborns, 1837 (60\%) were admitted in NICU and had some morbidity, out of which 398 $(13 \%)$ had Prematurity, 245 (8\%) had low birth weight, 306 (10\%) had Birth asphyxia, 214 (7\%) had neonatal sepsis, $153(5 \%)$ had pneumonia, 61 (2\%) had Diarrhoea, 31 (1\%) had congenital malformations, $214(7 \%)$ had jaundice, $92(3 \%)$ had Fits, $31(1 \%)$ were post term and $92(3 \%)$ had other morbidities.

Among the newborns admitted in NICUs of the selected military hospital 52\% were female and $48 \%$ were male. Out of the total 153 neonatal deaths in our study $53 \%$ were male and $47 \%$ were female.

Among the newborns admitted in NICUs with ${ }^{11}$ prematurity, $3.4 \%$ were newborns whose mothers were booked (had 4 or more ANC visits), whereas 96.6\% were newborns whose mothers had less than 4 ANC visits. 
Low birth weight, $6.4 \%$ were newborns whose mothers were booked (had 4 or more ANC visits), whereas $93.6 \%$ were newborns whose mothers had less than 4 ANC visits.

Birth Asphyxia, 12\% were newborns whose mothers were booked (had 4 or more ANC visits), whereas $88 \%$ were newborns whose mothers had less than 4 ANC visits.

Neonatal Sepsis, $12.3 \%$ were newborns whose mothers were booked (had 4 or more ANC visits), whereas $87.7 \%$ were newborns whose mothers had less than 4 ANC visits.

Pneumonia, $19.7 \%$ were newborns whose mothers were booked (had 4 or more ANC visits), whereas $80.3 \%$ were newborns whose mothers had less than 4 ANC visits.

Diarrhoea, $21.8 \%$ were newborns whose mothers were booked (had 4 or more ANC visits), whereas $78.2 \%$ were newborns whose mothers had less than 4 ANC visits.

Jaundice, $8 \%$ were newborns whose mothers were booked (had 4 or more ANC visits), whereas $92 \%$ were newborns whose mothers had less than 4 ANC visits.

Fits, 5.3\% were newborns whose mothers were booked (had 4 or more ANC visits), whereas $94.7 \%$ were newborns whose mothers had less than 4 ANC visits.

Mothers of all 7 newborns who were post term were unbooked.

The NMR of Pakistan in 2017 was 44.2 deaths per 1000 live births ${ }^{12,13}$. In our study, the NMR was 49.9 deaths per 1000 live births.

The frequency of prematurity ${ }^{14}, \mathrm{LBW}^{15}$, birth asphyxia ${ }^{16}$ has been reported to be lower in ANC users than those who did not attend ANC. Also ANC nonusers have a higher percentage (49\%) of anemia as compared to ANC users $(29 \%)^{17}$. Our study was consistent with these studies.

A study using secondary data recommends that in order to reduce neonatal mortality there is a need to implement interventions focusing on antenatal care effective referral system and retraining of health care providers to manage delivery complications and smaller than average birth size babies in resource-poor communities of Pakistan ${ }^{18}$. Our study also concluded that better use of ANC facilities can result in better pregnancy outcomes and can reduce neonatal mortality in Pakistan.
In Iran, the NMR in this Neonatal Intensive Care Unit was $6.44 / 1000$ during 7 years period ${ }^{19}$. The strong association between the mortality rate in the Neonatal Intensive Care Unit with preterm birth and low birth weight indicated the necessity of prenatal care aimed at preventing preterm labor ${ }^{20,21}$. Our study also showed that the neonatal mortality in booked mothers was less than one half of that in unbooked mothers.

\section{ACKNOWLEDGEMENT}

We are extremely grateful to Prof Dr Shahzad Ali Khan, HOD of Public Health at Health Services Academy Islamabad for his valuable input and kind guidance at every stage of this research. We extend our heartfelt gratitude to the administration and faculty of AFPGMI and all our seniors and colleagues for their support and cooperation.

\section{CONCLUSION}

In our study, it is concluded that not only the booked mothers in the selected military hospitals had better outcomes in terms of neonatal survival and health but neonatal health was strongly correlated with the number of antenatal visits of the mother. Neonatal morbidity and mortality can thus be prevented by increasing the utilization of existing antenatal care services, resulting in improved maternal, neonatal and perinatal health.

\section{CONFLICT OF INTEREST}

This study has no conflict of interest to be declared by any author.

\section{REFERENCES}

1. Gulland A. Children under 5 dying from preventable diseases, says UN. Br Med J 2016; 353.

2. UNICEF, WHO, World Bank, UN-DESA Population Division. UN Inter-agency Group for Child Mortality Estimation: Levels and Trends in Child Mortality: Estimates Developed by the UN Inter-agency Group for Child. UNICEF. 2014. Available from: http://www.unicef.org/media/files/Levels_and_Trends_in_Ch ild_Mortality_2014.pdf

3. United Nations Millennium Development Goals Report 2009. Available from: https://www.un.org/millenniumgoals/pdf/ MDG_Report_2009_ENG.pdf

4. Bellamy C. The state of the world's children: Childhood Under Threat. United Nations Children's Fund (UNICEF). 2005. Available from: https://www.unicef.org/sowc/archive/ English/The\%20State\%20of\%20the\%20World\%27s\%20Children \%202005.pdf

5. Imdad A, Salam R. Effects of neonatal nutrition interventions on neonatal mortality and child health and deve-lopment outcomes: a systematic review. Campbell Sys Rev 2019; 15(1-2).

6. Gupta J, Vegelin C. Sustainable development goals and inclusive development. Int Environ Agreements Polit Law Econ 2016; 16(3): 433-48. 
7. Kancherla V, Redpath B, Oakley GP. Reductions in child mortality by preventing spina bifida and anencephaly: Implications in achieving Target 3.2 of the Sustainable Development Goals in developing countries. Birth Defects Res 2019; 111(14): 958-66.

8. Oestergaard MZ. Neonatal mortality levels for 193 countries in 2009 with trends since 1990: A systematic analysis of progress, projections, and priorities. PLoS Med 2011; 8(8): e1001080.

9. Bhutta ZA, Hafeez A, Rizvi A. Reproductive, maternal, newborn, and child health in Pakistan: Challenges and opportunities. Lancet 2013; 381(9884): 2207-18.

10. Goli S, Arokiasamy P. Maternal and child mortality indicators across 187 countries of the world: Converging or diverging. Glob Public Health 2014; 9(3): 342-60.

11. Akbar R. Neonatal mortality and morbidity in booked and unbooked mothers in military hospitals (A comparative cross sectional study) [Thesis]. Rawalpindi, PK; AFPGMI: 2019.

12. Pakistan Demographic Health Survey PDHS 2018. Available from: http://catalog.ihsn.org/index.php/catalog/7970.

13. Nishtar S. Gateway Health Indicators of Pakistan - Gateway Paper II. Heartfile. 2007. Available from: http://www.heartfile. org/pdf/GWP-II.pdf

14. Neel S, Malhotra N, Swaminathan E, Neel PB, Lazar J, Gireesh $\mathrm{EV}$, et al. Study of integrated approach of antenatal care to improve the gestational age at birth. World J Anemia 2018; 2(1): 1-10.

15. Swain D. The incidence and factors of low birth weight among newborns with special reference to nursing practice in odisha - an observational study. Int J Obstet Perinat Neonatal Nurs 2017; 3(1): 26-32.

16. Wayessa ZJ, Belachew T, Joseph J. Birth asphyxia and associated factors among newborns delivered in Jimma zone public hospitals, Southwest Ethiopia: A cross-sectional study. J Midwifery Reprod Heal 2018; 6(2): 1289-95.

17. Tabinda Z, Khan S. Perinatal outcomes in antenatal service users versus non-users in an insured population of Northern Punjab, Pakistan. Pakistan J Public Heal 2012. Available from: https:// www.cabdirect.org/cabdirect/abstract/20133095756.

18. Nisar Y Bin, Dibley MJ. Determinants of neonatal mortality in Pakistan: Secondary analysis of Pakistan Demographic and Health Survey 2006-07. BMC Public Health 2014; 14(1): 1-5.

19. Hoseini B, Sadati MR. Assessment of neonatal mortality in the Neonatal Intensive Care Unit in Sabzevar City for the period of 2006-2013. Electron Physician 2015. Available from: https:// www.ncbi.nlm.nih.gov/pmc/articles/PMC4700895/

20. McClure EM, Goldenberg RL, Jobe AH, Miodovnik M, Thomas MK, Buekens P, et al. Reducing neonatal mortality associated with preterm birth: Gaps in knowledge of the impact of antenatal corticosteroids on preterm birth outcomes in low-middle income countries. Joao Paolo Souza. Reprod Health 2016; 13(1): 61-66.

21. Townsel C, Emmer SF, Campbell WA, Hussain N. Gender differences in respiratory morbidity and mortality of preterm neonates. Front Pediatr 2017; 5(1): 1-9. 